\title{
A concise classification of bencao (materia medica)
}

\author{
Zhongzhen Zhao*, Ping Guo* and Eric Brand
}

\begin{abstract}
Books that record the sources and applications of medicinal materials are commonly known as bencao (materia med$i c a$ ) in China. Bencao (materia medica) literature review is the very first step in the standard authentication procedure of Chinese medicinals. As an important part of China's cultural heritage, these various bencao (materia medica) texts represent centuries of accumulated wisdom in combating disease and preserving health. In this short review, bencao (materia medica) classics of China are broadly divided into three major categories in our routine practice: mainstream bencao (materia medica), thematic bencao (materia medica) and regional bencao (materia medica). The overall significance and current situation of exploration of bencao (materia medica) literature are summarized as well.
\end{abstract}

Keywords: Bencao (materia medica), Chinese medicinal authentication, Traditional Chinese medicine

\section{Background}

China is a large country with diverse ecological conditions and abundant botanical, zoological and mineral resources. Among them, some are of medicinal value and have been used medicinally since ancient times. In China, books that record the sources and applications of medicinal materials are commonly known as bencao (materia medica). The Chinese term "bencao", which literally means "rooted in herbs", reflects the fact that most medicinal materials are derived from botanical sources. Bencao (materia medica) texts of past dynasties primarily describe three aspects of Chinese medicinals: medicinal materials, medicinal properties, and medicinal principles. As an important part of China's cultural heritage, these various bencao (materia medica) texts represent centuries of accumulated wisdom in combating disease and preserving health. The Complete Collection of Traditional Texts on Chinese Materia Medica, a 410-volume and 246,000-page collection complied by the Association of Chinese Culture Research, includes more than 800 bencao (materia medica) classics from $220 \mathrm{BC}$ to 1911 $\mathrm{AD}$. This collection highlights the value of traditional

*Correspondence: zzzhao@hkbu.edu.hk; s193231@hkbu.edu.hk School of Chinese Medicine, Hong Kong Baptist University, Kowloon Tong, Hong Kong, China
Chinese medicine (TCM) as a rich source for knowledgebased medical rediscovery due to its documentation of clinical experiences over thousands of years, and also illustrates the monumental challenge of selecting the best parts of TCM for modern innovation ([1-3], Fig. 1).

The genre of bencao (materia medica) literature is uniquely developed in Chinese medicine, and represents a tremendous historical and cultural resource as well as an important reference point for clinicians, medical historians, and scientists in disciplines such as new drug discovery and Chinese medicinal authentication. Authentication is fundamental for Chinese medicinal standardization, and bencao (materia medica) literature review is the very first step in the standard authentication procedure of Chinese medicinals ([4], Fig. 2).

In practice, bencao (materia medica) classics of China are broadly divided into three major categories: (a) mainstream bencao (materia medica): the most influential bencao (materia medica) classics from key historical periods, (b) thematic bencao (materia medica): specialized bencao (materia medica) texts dedicated to specific topics, and c) regional bencao (materia medica): bencao (materia medica) texts focused on medicinal materials from specific regions.

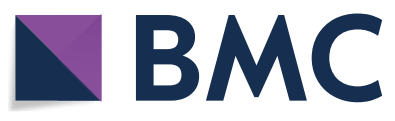

(c) The Author(s) 2018. This article is distributed under the terms of the Creative Commons Attribution 4.0 International License (http://creativecommons.org/licenses/by/4.0/), which permits unrestricted use, distribution, and reproduction in any medium, provided you give appropriate credit to the original author(s) and the source, provide a link to the Creative Commons license, and indicate if changes were made. The Creative Commons Public Domain Dedication waiver (http://creativecommons.org/ publicdomain/zero/1.0/) applies to the data made available in this article, unless otherwise stated. 


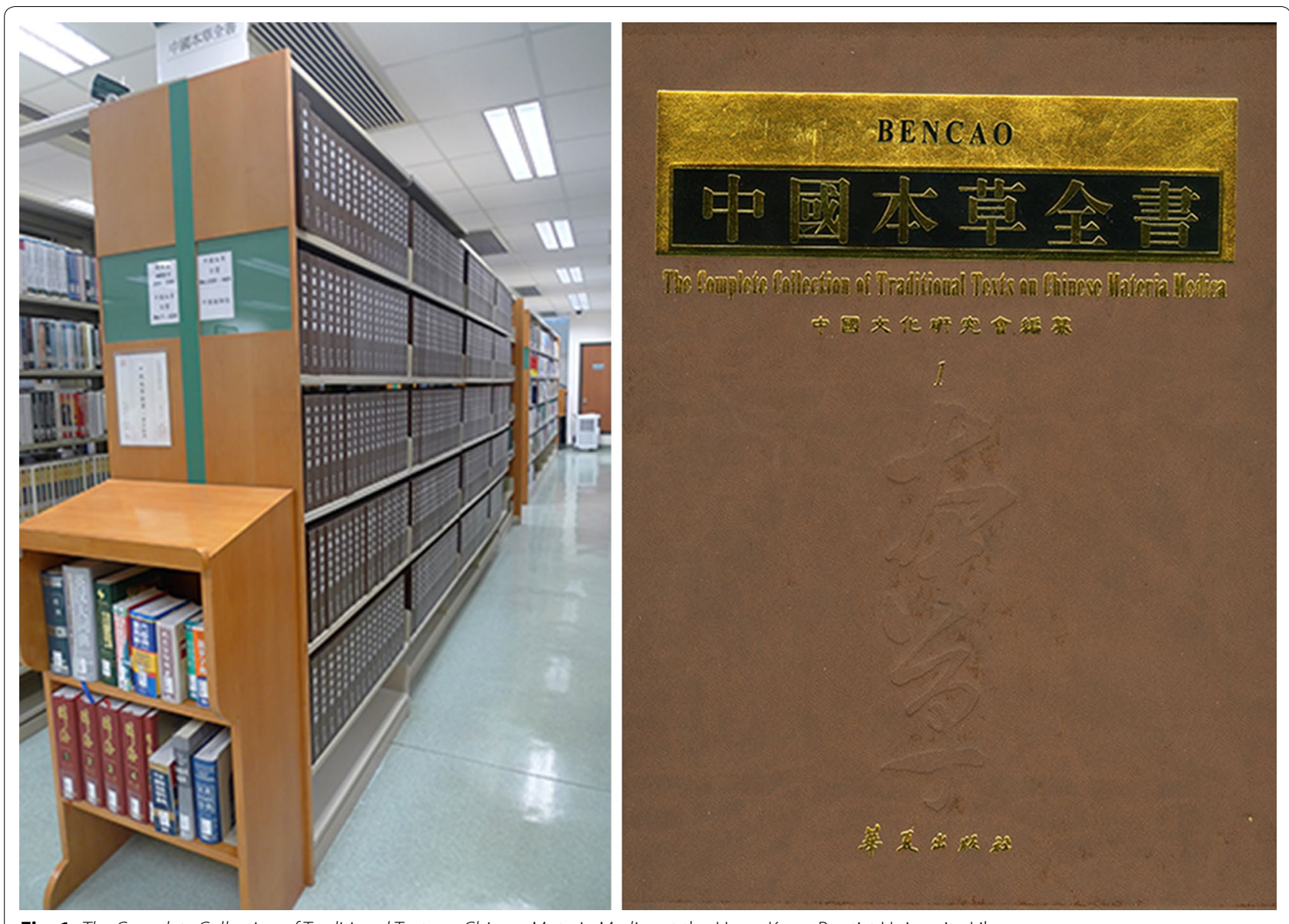

Fig. 1 The Complete Collection of Traditional Texts on Chinese Materia Medica at the Hong Kong Baptist University Library

\section{Mainstream bencao (materia medica)}

Over the past 2000 years, five monumental works stand out in the genre of bencao (materia medica) literature. They are the most influential classics from key historical periods.

(1) The Divine Husbandman's Classic of Materia Medica (Shen Nong Ben Cao Jing) This is the earliest extant bencao (materia medica) text, compiled in the Eastern Han Dynasty (25-220 AD). This text records 365 medicinals and summarizes medicinal experiences up to the Han Dynasty. Medicinals are classified into three categories (high-grade, medium-grade and low-grade) based on their medicinal effects and toxicity. Entries for each medicinal substance include nomenclature, properties, compatibilities, and medical applications. Descriptions of production regions and the ecological environment of some medicinal plants are also recorded briefly.

(2) Collection of Commentaries on the Classic of the Materia Medica (Ben Cao Jing Ji Zhu) Tao Hongjing, a physician of the North and South Kingdoms period (420-589 AD), compiled this text by preserving and annotating The Divine Husbandman's Classic of Materia Medica (Shen Nong Ben Cao Jing) and adding another 365 medicinals. It records 730 medicinals and established the framework of bencao (materia medica) compilations adopted by later generations. In this book, medicinal substances are further classified into seven categories based on their natural properties: jades/stones, herbs, trees, insects/beasts, fruits/vegetables, crops, and medicinals with names but without actual applications.

(3) Newly Revised Materia Medica (Xin Xiи Ben Cao) In $659 \mathrm{AD}$, commissioned by the government of the Tang Dynasty (618-907 AD), this text records 850 medicinals and is considered to be the earliest national pharmacopoeia in China.

(4) Materia Medica Arranged According to Pattern (Zheng Lei Ben Cao) Compiled by a physician named Tang Shenwei and published in 1108 $\mathrm{AD}$, this is the most praiseworthy bencao (materia medica) of the Song Dynasty (960-1279 AD) as it comprehensively summarizes herbal knowledge up 


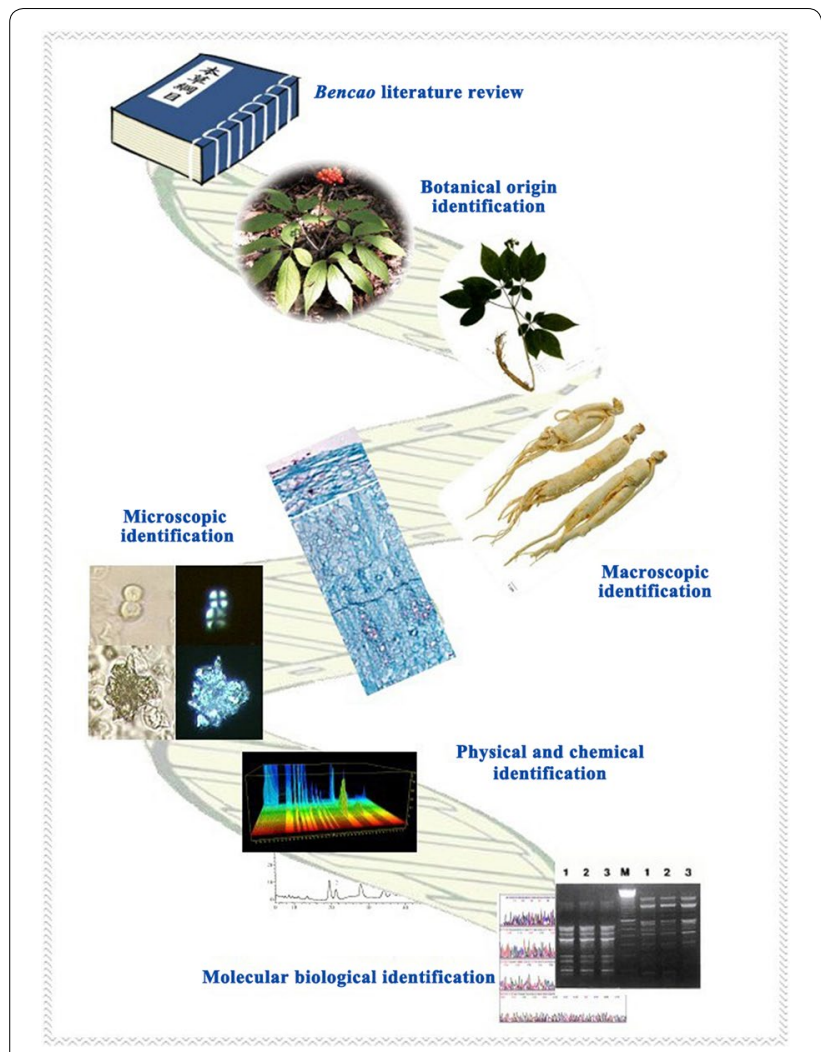

Fig. 2 Standard authentication procedure for Chinese medicinals

to that time. There are three versions of this book ("Da Guan", "Zheng He" and "Shao Xing") currently in circulation. It records 1746 medicinals and is the only bencao (materia medica) text from the Song and previous dynastic periods that survived intact. It features clearly preserved quotations from previous works and stands out as an important reference point for bencao (materia medica) knowledge leading up to the Song Dynasty.

(5) Compendium of Materia Medica (Ben Cao Gang $M u$ ) Written by Li Shizhen, a physician of the Ming Dynasty (1368-1644 AD), this text was first published in 1596. It records 1892 medicinal substances. This massive and influential compilation represents the highest academic achievement among all the ancient Chinese bencao (materia medica). It not only comprehensively summarized medical knowledge up to the sixteenth century in China, but also contributed greatly to the development of natural sciences in the world.

\section{Thematic bencao (materia medica)}

Thematic bencao (materia medica) refer to specialized bencao (materia medica) texts dedicated to specific topics, such as medicinal processing, authentication, dietary therapy, and medicinal properties. Examples include Grandfather Lei's Treatise on Herbal Processing (Lei Gong Pao Zhi Lun) and Origins of the Materia Medica (Ben Cao Yuan Shi). The former is the first monograph on Chinese medicinal processing, written in about 500 AD in the North and South Kingdoms period; it summarizes the literature and experiences of the ancient practice of processing. The latter stands out in the history of bencao (materia medica) as an outstanding monograph on macroscopic identification, written by Li Zhongli in the Ming Dynasty (1368-1644 AD). It is characterized by detailed illustrations and descriptions of diagnostic features of raw medicinal materials. Other examples of thematic bencao texts include the Materia Medica of Dietary Therapy (Shi Liao Ben Cao) of the Tang Dynasty and the Materia Medica for Decoctions (Tang Ye Ben Cao) of the Yuan Dynasty (1279-1368 AD). The former was dedicated to the theory and practice of dietary therapy, and the latter to the theory of medicinal properties and clinical experience.

\section{Regional bencao (materia medica)}

Regional bencao (materia medica) texts record knowledge related to medicinal substances derived from specific local regions. Examples include the Materia Medica from Steep Mountainsides (Lu Chan Yan Ben Cao) and the Essentials of Raw Herbs in Lingnan (Sheng Cao Yao Xing Bei Yao). The former was the first regional bencao (materia medica) with color illustrations dedicated to local medicinal plants; compiled in $1220 \mathrm{AD}$ in the Song Dynasty, it focused on the area around modernday Hangzhou. The latter was compiled in $1711 \mathrm{AD}$ in the Qing Dynasty (1644-1911 AD); it records botanical medicinals used in the Lingnan region, a geographic area located in the southern part of China.

\section{Conclusion}

Bencao (materia medica) tradition provides a rich record of knowledge that has been gradually refined for centuries, opening a window into the cultural tradition of scholarship and textual research that defines TCM. Bencao (materia medica) literature such as the Compendium of Materia Medica (Ben Cao Gang Mu) illustrates important developments in the broader history of natural sciences in China, and this text has been translated into European languages since 1735 AD. In recent decades, exploration of bencao (materia medica) literature has facilitated dramatic medical discoveries, such as the antimalarial drug artemisinin from sweet wormwood (qinghao, Artemisiae Annuae Herba) [5].

However, at present, the overall significance of bencao (materia medica) literature remains underestimated outside of the TCM community. Therefore, in addition to 
other aspects of TCM, attention is needed to preserve the cultural resources that lie at the heart of bencao (materia medica).

Although our research efforts related to daodi medicinal materials, medicinal authentication and Chinese medicinal processing are closely connected to bencao (materia medica) literature [6-9], the future of bencao (materia medica) research depends upon a multidisciplinary approach that protects the past while embracing the future.

\section{Abbreviation}

TCM: traditional Chinese medicine.

\section{Authors' contributions}

ZZ and PG designed the study. ZZ, PG and EB drafted and revised the manuscript. All authors read and approved the final manuscript.

\section{Acknowledgements}

Not applicable.

\section{Competing interests}

The authors declare that they have no competing interests.

\section{Availability of data and materials \\ Not applicable.}

\section{Consent for publication}

Not applicable.

\section{Ethics approval and consent to participate}

Not applicable.

\section{Funding}

Not applicable.

\section{Publisher's Note}

Springer Nature remains neutral with regard to jurisdictional claims in published maps and institutional affiliations.

Received: 10 February 2018 Accepted: 4 April 2018

Published online: 10 April 2018

\section{References}

1. Lu J, editor. The complete collection of traditional texts on Chinese materia medica. Beijing: Huaxia Publishing House; 1999.

2. Zhao ZZ, Liang ZT, Guo P, Chen HB. Medicinal plants of China. In: Singh RJ editor. Genetic resources, chromosome engineering, and crop improvement: medicinal plants, vol. 6. Boca Raton: CRC Press; 2012. p. 123-62.

3. Xu QH, Bauer R, Hendry BM, Fan TP, Zhao ZZ, Duez P, Simmonds MSJ, Witt CM, Lu AP, Robinson N, Guo DA, Hylands PJ. The quest for modernisation of traditional Chinese medicine. BMC Complement Altern Med. 2013;13:132.

4. Zhao ZZ, Hu YN, Liang ZT, Yuen JPS, Jiang ZH, Leung KSY. Authentication is fundamental for standardization of Chinese medicines. Planta Med. 2006;72:865-74.

5. Tu YY. The discovery of artemisinin (qinghaosu) and gifts from Chinese medicine. Nat Med. 2011;17:1217-20.

6. Zhao ZZ, Guo P, Brand E. The formation of daodi medicinal materials. J Ethnopharmacol. 2012;140:476-81.

7. Zhao ZZ, Liang ZT, Guo P. Macroscopic identification of Chinese medicinal materials: traditional experiences and modern understanding. J Ethnopharmacol. 2011;134:556-64.

8. Zhao ZZ, Chen HB, Guo P, Brand E. Chinese medicinal identification: an illustrated approach. Taos: Paradigm Publications; 2014

9. Guo P, Brand E, Zhao ZZ. Chinese medicinal processing: a characteristic aspect of ethnopharmacology of traditional Chinese medicine. In: Heinrich M, Jager AK, editors. ethnopharmacology. Chichester: Wiley; 2015. p. 303-16.
Ready to submit your research? Choose BMC and benefit from:

- fast, convenient online submission

- thorough peer review by experienced researchers in your field

- rapid publication on acceptance

- support for research data, including large and complex data types

- gold Open Access which fosters wider collaboration and increased citations

- maximum visibility for your research: over $100 \mathrm{M}$ website views per year

At BMC, research is always in progress.

Learn more biomedcentral.com/submissions 